\title{
ODONTOLOGÍA BASADA EN EVIDENCIA: CONCEPTOS GENERALES Y SU RELEVANCIA
}

\section{EVIDENCE-BASED DENTISTRY: RELEVANCE AND GENERAL CONCEPTS}

\author{
Andrés Viteri-García ${ }^{1,3}$, Nadia Montero ${ }^{1,3}$, Ingrid Arévalo-Rodríguez ${ }^{1,3}$, Ana Armas-Vega ${ }^{2,3}$, Camilo Félix ${ }^{3}$, Daniel \\ Simancas-Racines ${ }^{1,3}$
}

\section{Resumen}

La Odontología Basada en la Evidencia (OBE) es un concepto ampliamente difundido y reconocido en las ciencias de la salud. Ésta busca que las decisiones en el campo odontológico se basen en datos válidos y confiables de investigaciones desarrolladas con criterios de calidad, las cuales, junto con la experiencia del profesional y las preferencias de los pacientes, se conviertan en la mejor opción para la atención integral y de calidad. El objetivo de esta revisión es resumir los principi os de la Odontología Basada en la Evidencia. Se realizó una búsqueda manual de la literatura relevante y se presentan los conceptos y principios de la OBE, así como ejemplos de las fases de la misma desde la articulación de la pregunta PICO, pasando por la búsqueda de información y lectura crítica para finalmente llegar a la aplicación de estos conocimientos en nuestros pacientes. Se concluye que la OBE permite emplear la mejor evidencia científica sin dejar de lado la experiencia, juicio profesional y preferencias de los pacientes en la toma de decisiones en la práctica odontológica diaria.

Palabras clave: Odontología Basada en la Evidencia, Práctica Clínica Basada en la Evidencia

\begin{abstract}
The Evidence-Based Dentistry (EBD) is a worldwide-recognized concept in the health sciences. It seeks to make clinical decisions based on reliable data from well-conducted researches, which with professional's experience and patient's preferences become the best option that can be offered to them. The aim of this review is to summarize the principles of EBD. Hand search of relevant literature was made, OBE concepts and principles are shown as well as examples of the formulation of the PICO question, the search and critical appraisal of information, and the application of that knowledge to ou r patients. It is concluded that the EBD allows the use of the best scientific evidence without leaving aside the experience, professional judgment and patient's preferences in the decision-making of the daily dentistry practice.
\end{abstract}

Key words: Evidence-Based Dentistry, Evidence-Based Practice

${ }^{1}$ Centro de Investigación en Salud Pública y Epidemiología Clínica (CISPEC).

${ }^{2}$ Centro de Investigación en Salud Oral (CISO).

${ }^{3}$ Facultad de Ciencias de la Salud Eugenio Espejo. Universidad Tecnológica Equinoccial. Quito-Ecuador.

\section{Correspondencia:}

Andrés Viteri García DDS.

Dirección: Av. Mariscal Sucre s/n y Av. Mariana de Jesús. Quito, Ecuador. Teléfonos: 2990800 ext. 2165

Correo electrónico: andres.viteri@ute.edu.ec.
Este es un artículo Open Access distribuido bajo la licencia Creative Commons Atribución-NoComercialCompartir Igual 4.0

\section{두()ㅇㅇㄱ}

Citar como: Viteri- García A, Montero N, Arévalo - Rodríguez I, Armas -Vega A, Félix C, Simancas - Racines D. Odontología basada en evidencia: conceptos generales y su relevancia. KIRU. 2018; 15(1): 55 - 61. https://doi.org/10.24265/kiru.2018.v15n1.07 


\section{INTRODUCCIÓN}

La odontología basada en evidencia (OBE o EBD por sus siglas en inglés), es una estrategia que buscaque las decisiones clínicas diarias que toma el profesional odontólogo estén basadas en la evidencia científica (1, 2). La idea de la necesidad de mejorar la práctica clínica con base a lo que hoy conocemos como método científico, fue propuesta a partir de los experimentos realizados por Louis hace más de 300 años (3), pero fue en 1990 que Gordon Guyatt finalmente acuñó el término Evidence-based Medicine, y en 1996 que David Sackett definió la Medicina Basada en Evidencia (MBE) como "la utilización consciente, explícita y juiciosa de la mejor certeza científica, para tomar decisiones relacionadas con el cuidado de la salud del paciente" (4).

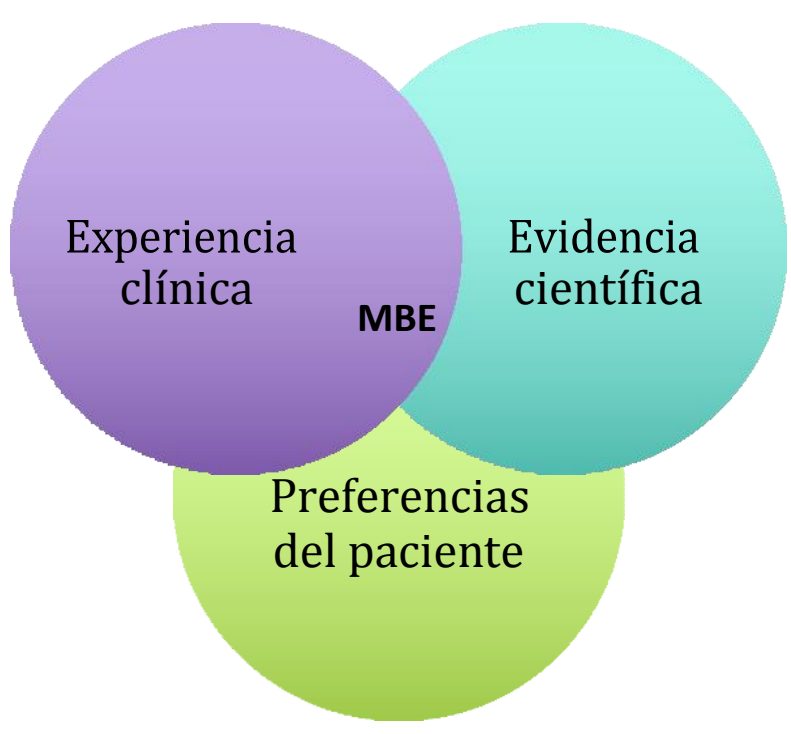

Figura 1. La triada de la MBE Según lo propuesto por Sackett y col. (4)

Desde entonces, éste concepto ha evolucionado para incluir la experiencia clínica, la mejor evidencia publicada en la literatura científica y las preferencias del paciente en la toma de decisiones sobre problemas clínicos de toda índole. En la actualidad, el concepto de la práctica basada en la evidencia (PBE) es entonces aplicable a todas las ciencias de la salud, incluyendo a la odontología. A continuación, explicaremos/ presentaremos el concepto de MBE aplicado a este campo, así como ejemplos de cómo éste enfoque puede ser empleado en la toma de decisiones para mejorar la atención odontológica a los pacientes.

\section{El proceso de la OBE}

La Odontología Basada en Evidencia corresponde a un proceso sistemático para buscar información y aplicarla al contexto clínico odontológico, la cual puede resumirse en los siguientes pasos: 1 . Identificación del problema clínico, 2. Transformación de las dudas clínicas en preguntas contestables (Estrategia PICO), 3. Identificación del mejor tipo de estudios para contestar la pregunta o preguntas seleccionadas, 4. Búsqueda de la mejor evidencia disponible para responder la pregunta, 5. Lectura crítica de la evidencia disponible, y finalmente, 6 . Aplicación de los resultados, integrando la mejor evidencia disponible con la experiencia clínica y preferencias del paciente.

\section{Identificación del problema clínico}

Este paso consiste en identificar y/o jerarquizar las dudas clínicas que surgen dentro del contexto de la atención a un determinado paciente, priorizando las más urgentes o importantes, las mismas que deberán ser resueltas para garantizar el bienestar de los pacientes. Estas dudas pueden surgir en cualquier momento de la atención, por ejemplo, al prescribir una prueba diagnóstica, indicar un determinado tratamiento, o planear un seguimiento, etc.

Por ejemplo, en la atención a un paciente que consulta por dolor luego de la extracción quirúrgica de terceros molares, el odontólogo puede tener en dudas sobre qué analgésico antiinflamatorio prescribir: Paracetamol $1 \mathrm{~g}$ ○ Ibuprofeno $800 \mathrm{mg}$, teniendo en cuenta la eficacia y efectos adversos comparados de las dos intervenciones en el manejo del dolor post quirúrgico. También pueden surgir dudas cómo ¿qué pruebas diagnósticas son indispensables para el correcto diagnóstico, o estadificación de una condición?; por ejemplo, en un paciente que presenta caries inter proximal, se puede dudar entre prescribir la realización de una radiografía peri apical o de aleta de mordida (bite wing). Por esta razón es necesario estandarizar las dudas clínicas en preguntas contestables.

2. Transformación de las dudas clínicas en preguntas contestables

Una vez establecida la(s) duda(s) clínica(s), es necesario transformarla(s) en una/varias preguntas(s) definidas de manera simple y clara. Para este fin se utiliza la estrategia PICO, que hace referencia al acrónimo de los elementos que debemos considerar para formular una pregunta bien construida: $\mathrm{P}=$ Paciente, $\mathrm{I}=$ Intervención, $\mathrm{C}=$ Comparación y $\mathrm{O}=$ Desenlace (Outcome por su término en inglés). 
Para un caso hipotético donde un paciente de 55 años saludable que presenta periodontitis crónica, el cual acude a consulta en busca de tratamiento, manifiesta temor a las intervenciones odontológicas quirúrgicas, por lo que desea pasar el menor tiempo posible en consulta, así como resolver los signos y síntomas de la periodontitis (5). Una de las preguntas que puede formularse mediante la estrategia PICO se presenta en la tabla 1.

Tabla 1. Ejemplo de pregunta PICO

\begin{tabular}{|c|c|}
\hline P (Paciente) & Adulto con periodontitis crónica \\
\hline (Intervención) & $\begin{array}{l}\text { Tratamiento periodontal con equipo de } \\
\text { ultrasonido }\end{array}$ \\
\hline $\begin{array}{l}\text { C } \\
\text { (Comparación) }\end{array}$ & $\begin{array}{l}\text { Tratamiento periodontal con } \\
\text { instrumental manual }\end{array}$ \\
\hline $\begin{array}{l}\text { O } \\
\text { (Desenlaces) }\end{array}$ & $\begin{array}{l}\text { Efectividad (medida por la } \\
\text { adherencia al tratamiento), } \\
\text { reducción de la bolsa (medida al } \\
\text { sondaje periodontal), eficiencia } \\
\text { (medida por el tiempo del } \\
\text { tratamiento). } \\
\text { Seguridad (no evaluada) }\end{array}$ \\
\hline
\end{tabular}

*Parcialmente tomada del estudio de Faggion y colaboradores ${ }^{(5)}$

Así, la pregunta sería: En pacientes adultos con periodontitis crónica, ¿qué tratamiento no quirúrgico (con equipos ultrasónicos o con instrumentos manuales) es más efectivo y seguro para el manejo de la periodontitis crónica, en términos de la reducción de la bolsa al sondaje periodontal, la adherencia al tratamiento, y la duración del mismo? (5).

\section{Identificación del tipo de estudios que mejor} contesten la pregunta

Existen diferentes tipos de estudios los cuales resultan relevantes en el campo de la odontología. Sin embargo, no todos contestan el mismo tipo de

preguntas. Para poder direccionar nuestra búsqueda a aquellos que serán más relevantes para la pregunta que nos hemos planteado, es necesario reconocer los diferentes diseños de investigación en salud. En la tabla 2 se muestran dichos diseños con ejemplos aplicados al campo de la salud oral.

Para el ejemplo propuesto (manejo no quirúrgico de la periodontitis), es necesario utilizar ensayos clínicos aleatorizados controlados, así como revisiones sistemáticas de ensayos clínicos, ya que corresponde a una pregunta relacionada con el tratamiento/abordaje de una patología específica. Este tipo de preguntas nos permite comparar al menos dos grupos de pacientes con condiciones basales similares, de tal manera que sólo se diferencien por el tratamiento que reciben para el manejo de la condición de estudio. Este diseño de estudios establece un seguimiento para los desenlaces clínicos más

Tabla 2. Diseños de investigación clínica.

\begin{tabular}{lll}
\hline Pregunta orientada a: & Tipo de estudio más frecuente & Ejemplo
\end{tabular}

\begin{tabular}{|c|c|c|}
\hline Diagnóstico & $\begin{array}{c}\text { De corte transversal/ De pruebas } \\
\text { diagnósticas }\end{array}$ & $\begin{array}{l}\text { P: Pacientes con caries } \\
\text { I: Inspección visual-táctil (ICDAS, CPOD) } \\
\text { C: Fluorescencia láser (Diagnodent) } \\
\text { O: Precisión en el diagnóstico de caries (6) }\end{array}$ \\
\hline $\begin{array}{l}\text { Pronóstico/ } \\
\text { Riesgo/ } \\
\text { Etiología }\end{array}$ & Cohortes & $\begin{array}{l}\text { P: Adultos mayores } \\
\text { I: Presencia de periodontitis } \\
\text { C: Periodontalmente sanos } \\
\text { O: Desarrollo de Demencia (7) }\end{array}$ \\
\hline $\begin{array}{l}\text { Prevención/ } \\
\text { Tratamiento }\end{array}$ & $\begin{array}{l}\text { Ensayos clínicos aleatorizados y } \\
\text { controlados/Revisiones } \\
\text { sistemáticas (de intervención) }\end{array}$ & $\begin{array}{l}\text { P: Adultos con periodontitis crónica } \\
\text { I: Tratamiento con equipo de ultrasonido } \\
\text { C: Tratamiento con instrumental manual } \\
\text { O: Resolución de los síntomas (5) }\end{array}$ \\
\hline
\end{tabular}

Fuente: Parcialmente tomada del estudio de Simancas y colaboradores ${ }^{(8)}$ 
relevantes, y al final evalúa a los pacientes en relación a criterios predefinidos de efectividad y seguridad $(9,10)$.

4. Búsqueda de la mejor evidencia disponible para responder la pregunta clínica

La literatura científica se encuentra jerarquizada de acuerdo a la calidad de información y metodología empleada para obtenerla/analizarla (figura. 2). Es importante que tratemos siempre de encontrar las respuestas a nuestras dudas en los niveles más altos de dicha jerarquía, lo cual no sólo nos asegurará mayor calidad de información, sino que también, en muchas ocasiones, nos ahorrará tiempo en la búsqueda de la misma.

Las revisiones sistemáticas de la literatura científica constituyen una herramienta esencial para sintetizar la información científica disponible, incrementar la validez de las conclusiones de estudios individuales e identificar áreas de incertidumbre donde sea necesario realizar investigación (12). Son imprescindibles para una práctica basada en la evidencia y una herramienta fundamental en la toma de decisiones en las ciencias de la salud (13).

Al realizar una búsqueda están a disponibilidad de los investigadores varios motores de búsqueda y bases de datos que identifican y/o recopilan información científica ${ }^{(14)}$. En la tabla No. 3 se resumen aquellas que son relevantes en el campo de la odontología y se les otorga una breve descripción.

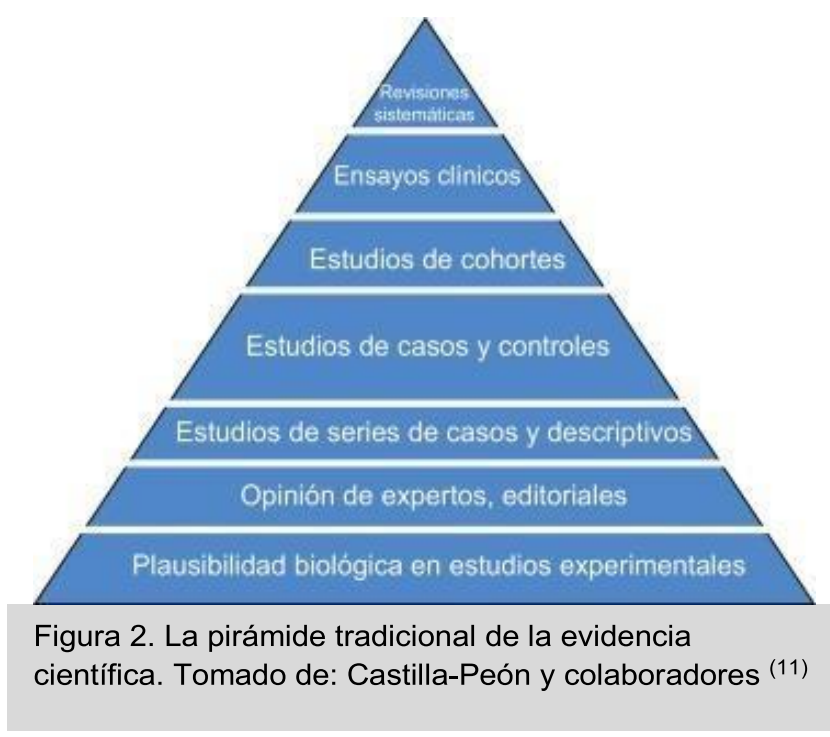

Tabla 3. Motores de búsqueda, bases de datos y revistas en odontología

\begin{tabular}{|c|c|c|}
\hline Descripción & Institución & Enlaces \\
\hline \multirow[t]{4}{*}{ Motores de Búsqueda } & MEDLINE (PubMed) & http://www.ncbi.nlm.nih.gov/pubmed/ \\
\hline & $\begin{array}{c}\text { EBSCO (Oral Health Sciences } \\
\text { Source) }\end{array}$ & $\begin{array}{c}\text { https://www.ebscohost.com/academic/dentistr } \\
\text { y-oral-sciences-source }\end{array}$ \\
\hline & LILACS & http://lilacs.bvsalud.org \\
\hline & Embase & https://www.embase.com/login \\
\hline Meta buscadores & TRIP Database & http://www.tripdatabase.com \\
\hline \multirow{4}{*}{$\begin{array}{c}\text { Centros } \\
\text { desarrolladores de } \\
\text { literatura integrativa }\end{array}$} & The Cochrane Library & http://www.cochranelibrary.com \\
\hline & $\begin{array}{l}\text { Scottish Intercollegiate } \\
\text { Guidelines Network }\end{array}$ & http://www.sign.ac \\
\hline & $\begin{array}{l}\text { National Institute for Health } \\
\text { and Care Excellence }\end{array}$ & http://www.nice.org.uk \\
\hline & $\begin{array}{l}\text { New Zealand Guidelines } \\
\text { Group }\end{array}$ & http://www.nzgg.org.nz \\
\hline
\end{tabular}




\begin{tabular}{|c|c|c|}
\hline & $\begin{array}{l}\text { Institute for Clinical System } \\
\text { Improvement }\end{array}$ & http://www.icsi.org \\
\hline \multirow[t]{10}{*}{$\begin{array}{l}\text { Centros y revistas de } \\
\text { investigación } \\
\text { odontológica }\end{array}$} & $\begin{array}{l}\text { International Association for } \\
\text { Dental Research (Journal of } \\
\text { Dental Research) }\end{array}$ & $\begin{array}{l}\text { http://www.iadr.com/i4a/pages/index.cfm?pag } \\
\qquad \text { eid=1 } \\
\text { http: //journals.sagepub.com/home/jdr }\end{array}$ \\
\hline & $\begin{array}{c}\text { Journal of Applied Oral } \\
\text { Science }\end{array}$ & http://www.revistas.usp.br/jaos \\
\hline & Brazilian Oral Research & $\begin{array}{l}\text { https://www.researchgate.net/journal/1806- } \\
\text { 8324_Brazilian_Oral_Research }\end{array}$ \\
\hline & $\begin{array}{l}\text { Journal of Clinical and } \\
\text { Experimental Dentistry }\end{array}$ & http://www.medicinaoral.com/odo/indice.htm \\
\hline & $\begin{array}{l}\text { International Endodontic } \\
\text { Journal }\end{array}$ & $\begin{array}{l}\text { http://onlinelibrary.wiley.com/journal/10.1111/( } \\
\text { ISSN)1365-2591 }\end{array}$ \\
\hline & $\begin{array}{l}\text { Journal of Clinical } \\
\text { Periodontology }\end{array}$ & $\begin{array}{l}\text { http://onlinelibrary.wiley.com/journal/10.1111/( } \\
\text { ISSN)1600-051X }\end{array}$ \\
\hline & Oral Oncology & $\begin{array}{c}\text { https://www.journals.elsevier.com/oral- } \\
\text { oncology/ }\end{array}$ \\
\hline & $\begin{array}{l}\text { Clinical Oral Implants } \\
\text { Research }\end{array}$ & $\begin{array}{l}\text { http://onlinelibrary.wiley.com/journal/10.1111/( } \\
\text { ISSN)1600-0501 }\end{array}$ \\
\hline & $\begin{array}{l}\text { Journal of Cranio-Maxillo- } \\
\text { Facial Surgery }\end{array}$ & http://www.jcmfs.com \\
\hline & Angle Orthodontist & http://www.angle.org/?code=angf-site \\
\hline
\end{tabular}

Realizada por los autores

5. Lectura crítica de la evidencia disponible

Una vez identificadas las referencias relevantes para la pregunta clínica, el siguiente paso es determinar su validez, es decir el grado de certeza de que el desenlace estudiado se acerque a la realidad, así como su utilidad, es decir su aplicabilidad en el escenario clínico propuesto. Para este fin se han desarrollado varias herramientas como las guías de JAMA (15), las preguntas de evaluación crítica de la Red CASPe y la evaluación del Riesgo de Sesgo (Risk of Bias) de la colaboración Cochrane $(8,16)$.

Por ejemplo, en la revisión sistemática titulada "
"Aislamiento con dique de goma para tratamientos restauradores en pacientes odontológicos" los autores de la revisión usaron la herramienta para riesgo de sesgo de la Colaboración Cochrane, y encontraron que los cuatro estudios que cumplieron con los criterios de inclusión establecidos tenían alto riesgo de sesgo, debido a la falta de cegamiento de los participantes y evaluadores al tratamiento, y tres de ellos debido a sesgo de reporte (reporting bias) ${ }^{(17)}$.

Por tanto, los hallazgos numéricos de dichos estudios podrían no ser correctos. Sin la evaluación de este aspecto, los clínicos podrían pensar que el aislamiento 
absoluto es el tratamiento ideal, mientras que la evidencia nos muestra que existen problemas en la adopción de los resultados de dichos estudios. Hallazgos como este nos permiten entender la importancia de la lectura crítica

\section{Aplicación de los resultados}

Una vez se ha obtenido la información derivada de la evidencia científica, la cual se ha valorado en su calidad y cantidad, se debe tomar en cuenta las características propias del entorno de aplicación, las variables socio-demográficas, culturales, de costos, y de preferencias de nuestros pacientes, a fin de valorar la aplicación de la evidencia al contexto clínico en el cual surgió la pregunta clínica seleccionada. Estos aspectos son de marcada importancia al momento de extrapolar los hallazgos obtenidos en nuestra búsqueda a la práctica diaria. Algunas preguntas que nos pueden ayudar en este proceso son:

- ¿Pueden aplicarse estos resultados en mis pacientes? Por tanto, en este punto se deben considerar cualquier factor que impida que la evidencia identificada pueda trasladarse al contexto clínico propio. Esto puede ser por diferencias en el nivel de atención, la población atendida, las comorbilidades del paciente, etc.

- ¿Se tuvieron en cuenta todos los resultados de importancia clínica? En este caso, se debe enfatizar en los resultados que son relevantes para el paciente en el contexto clínico propio, los cuales pueden diferir de los que presenta la evidencia recopilada.

- ¿Los beneficios a obtener justifican los riesgos y/o costes ${ }^{(8)}$. En este caso, se deben balancear los costos del uso de las diferentes opciones de manejo evaluadas (en caso de preguntas de intervenciones), con los posibles eventos adversos que se derivan de su administración.

Por ejemplo, para un caso en el que un paciente requiere analgesia para controlar el dolor postquirúrgico, después de realizar el proceso de la OBE usted encuentra que no existe diferencia entre ketorolaco cuando es comparado con otros AINES en control del dolor (18) y usted como odontólogo decide que la administración de ketorolaco es lo indicado (30mg/1ml IM o $10 \mathrm{mg} \mathrm{VO}$ ), es muy probable que el paciente prefiera que la misma sea vía oral en lugar de vía intramuscular, y por tanto la decisión deberá considerar la experiencia clínica del profesional y las preferencias de los pacientes.
En el caso del manejo de la periodontitis crónica por vías no quirúrgicas la evidencia muestra que no existe diferencia significativa entre los desenlaces clínicos propuestos para el tratamiento con equipo de ultrasonido cuando es comparado con tratamiento con instrumental manual $(5,19)$, sin embargo el uso de equipo ultrasónico disminuye el tiempo de tratamiento, por lo que sería la opción que más interesaría a nuestro paciente, debido a que ha expuesto que su interés es pasar el menor tiempo posible en consulta.

\section{CONCLUSIONES}

El acelerado avance de las innovaciones científicas y tecnológicas en el campo odontológico hace necesario un cambio en la práctica clínica diaria. LaOdontología Basada en Evidencia es una estrategia que, a través de la elaboración de la pregunta PICO, la búsqueda de información y la lectura crítica, permite aplicar la mejor evidencia científica sin dejar de lado la experiencia, juicio profesional y preferencias de los pacientes. Como todos los profesionales en salud, el profesional odontólogo tiene la responsabilidad de adecuar su accionar clínico con la mejor evidencia disponible, dejando de lado la práctica basada en el empirismo, no solo para proveer el mejor tratamiento a su paciente, sino para mejorar la calidad asistencial en general, así como fomentar la educación y actualización continua propia y de sus colegas.

\section{Conflictos de interés}

Los autores declaran no presentar ningún conflicto de interés.

\section{Agradecimientos}

A Solange Núñez y Diana Buitrago por la revisión y comentarios de este artículo.

\section{REFERENCIAS BIBLIOGRÁFICAS}

1. Lawrence R. Evidence based dentistry. British dental journal. 1995; 179(7): 270-3.

2. Brignardello-Petersen RC; Glick A.; Guyatt G.; Azarpazhooh A. A practical approach to evidencebased dentistry: Understanding and applying the principles of EBD. The Journal of The American Dental Association. 2014;145(11)

3. Morabia A. P. C. A. Louis and the birth of clinical epidemiology. Journal of clinical epidemiology. 1996;49(12):1327-33.

4. Sackett DL; Rosenberg WM; Gray JA; Haynes RB; Richardson WS. Evidence based medicine: what it is and what it isn't. BMJ (Clinical research ed). 1996;312(7023):71-2. 
5. Faggion CM Jr; Tu YK. Evidence-based dentistry: a model for clinical practice. Journal of dental education. 2007;71(6):825-31.

6. Iranzo-Cortes JT S; Montiel-Company J; Almerich-Silla J. Diagnosis validity of ICDAS and DIAGNOdent combined: an in vitro stdy in pre-cavitated lesions. Lasers in medical science. 2017;32(3):543-8

7. Lee Y TLH C; Hu CJ; Huang LK. Chao SP; Lin CP; Su ECY; Lee YC; Chen CC. Periodontitis as a Modifiable Risk Factor for Dementia: A Nationwide Populationbased Cohort Study. Journal of the American Geriatrics Society. 2017;65(2):301-5.

8. Simancas-Racines DS; Pinos ML. Evidence Based Medicine. General concepts and it's importance in Dentistry. Tsafiqui. 2014;14(3):145-51.

9. WHO. Healthtopics. Clinical trials. 2017. Disponible en la web: http://www.who.int/topics/clinical_trials/en/

10. Sibbald B; Roland M. Understanding controlled trials. Why are randomised controlled trialsimportant?. BMJ: British Medical Journal. 1998;316(7126):201.

11. Castilla-Peón MF; Ramírez-Sandoval JC; ReyesMorales H; Reyes-López A. Diseño de estudios clínicos y causalidad: ¿la vacuna oral contra rotavirus causa invaginación intestinal?. Boletín Médico del Hospital Infantil de México. 2015;72(5):346-52.

12. Ferreira Gonzalez IU; Alonso-Coello P. Revisiones sistemáticas y metaanálisis: bases conceptuales e interpretación. Revista Española de Cardiología. $2011 ; 64(08): 688-96$
13. Arevalo-Rodriguez I. ¿Qué es la literatura integrativa?. Conferencia presentación CISPEC-UTE. 2017.

14. Brignardello-Petersen R; Carrasco-Labra A; Booth HA;

Glick M; Guyatt GH; Azarpazhooh A; et al. A practical approach to evidence-based dentistry: How to search for evidence to inform clinical decisions. Journal of the American Dental Association. 2014;145(12):1262-7.

15. Oxman AD, Sackett DL; Guyatt GH. Users' guides to the medical literature. I. How to get started. The EvidenceBased Medicine Working Group. Jama. 1993;270(17):2093-5.

16. Bonfil X. La Colaboración Cochrane. Jano. 1997;52(1204):63-5.

17. Wang Y; Li C; Yuan H; Wong MCM; Zou J; Shi Z; et al. Rubber dam isolation for restorative treatment in dental patients. Cochrane Database of Systematic Reviews. 2016(9).

18. CADTH. Ketorolac for Pain Management: A Review of the Clinical Evidence. Canadian Agency for Drugs and Technologies in Health ACDTH. 201430 June 2014.

Disponible en la web:
https://www.cadth.ca/sites/default/files/pdf/htis/nov2014/RC0565Toradol Final.pdf

19. Tunkel J; Heinecke A; Flemmig TF. A systematic review of efficacy of machine-driven and manual subgingival debridement in the treatment of chronic periodontitis. Journal of clinical periodontology. 2002;29 Suppl 3:7281; discussion 90-1. 